\title{
Artrocentesis temporomandibular: Un tratamiento para la abertura bucal limitada con dolor agudo
}

Arthrocentesis of the temporomandibular joint: a treatment for the acute-pain limited mouth opening (using a new device)

\section{Resumen}

La Artrocentesis de la articulación temporomandibular (ATM) es una alternativa de tratamiento eficaz; mínimamente invasiva para la abertura bucal limitada debido al desarreglo interno discal irreductible o al deterioro de las estructuras de la ATM que producen artralgia severa que no cede con tratamientos conservadores. Se presenta un caso clínico de una paciente con limitación de abertura y dolor orofacial intenso que se solucionó satisfactoriamente con lavaje articular con un nuevo dispositivo de una sola punción. Se mejora la abertura bucal con el consiguiente alivio del dolor y satisfacción del paciente en una sola sesión. Utilizando este dispositivo, no se requiere la inserción de dos agujas; lo que facilita, el procedimiento y lo hace menos invasivo.

Palabras clave: Artrocentesis temporomandibular; trastornos temporomandibulares, abertura bucal limitada.

Abstract

La Artrocentesis de la articulación temporomandibular (ATM) es una alternativa de tratamiento eficaz; mínimamente invasiva para la abertura bucal limitada debido al desarreglo interno discal irreductible o al deterioro de las estructuras de la ATM que producen artralgia severa que no cede con tratamientos conservadores. Se presenta un caso clínico de una paciente con limitación de abertura y dolor orofacial intenso que se soluciono satisfactoriamente con lavaje articular con un nuevo dispositivo de una sola punción. Se mejora la abertura bucal con el consiguiente alivio del dolor y satisfacción del paciente en una sola sesión. Utilizando este dispositivo, no se requiere la inserción de dos agujas; lo que facilita, el procedimiento y lo hace menos invasivo.

Key words: Arthrocentesis of the temporomandibular joint; temporomandibular disorders, limited mouth opening.

\section{Introducción}

Se denomina limitación de la abertura de la boca cuando se presenta la imposibilidad de abrir la boca no más de 25 $\mathrm{mm}$. Los intentos de abrir la boca más amplia producen dolor pre-auricular o en los músculos masticatorios. ${ }^{1}$

La limitación en la abertura de la boca puede ser el resultado de procesos patológicos, ya sea en la ATM (intra-articulares) o de los tejidos extra-articulares del sistema masticatorio. Las causas comunes de la abertura bucal limitada, son las asociadas con trastornos intraarticulares de la ATM como el desplazamiento del disco sin reducción, o el síndrome de disco fijo, o la enfermedad degenerativa con dolor en las articulaciones (artralgia temporomandibular). ${ }^{2}$

El disco de la ATM cuando está desplazado anteromedialmente o esta "pegado" al compartimiento superior, impide los movimientos de traslación del cóndilo mandibular a lo largo de la pendiente posterior de la eminencia articular, por lo tanto, solo permite hacer rotación condilar y esta condición limita súbita o progresivamente la abertura de la boca. Los músculos de la masticación no están afectados y la abertura forzada de la boca produce dolor en la zona retrodiscal bilaminar de la articulación afectada. ${ }^{3,4}$

En la imagenología ATM: las radiografías panorámicas se pueden, observar alteraciones o remodelaciones de la morfología condilar; la radiografia trascraneal de ATM evidencia la traslación condilar y la tomografía computarizada mostraría, con detalle, los cambios morfologícos articulare. $s^{5}$ Solo las imágenes de resonancia magnética nos permiten visualizar si el disco está desplazado o "pegado" a la eminencia articular. Se ha postulado que la patogénesis de esta condicion se debe a la alteración de la lubricación en la articulación. ${ }^{6}$

El desarrollo de la artrocentesis para la articulación temporo-mandibular (ATM) surge como consecuencia de los hallazgos que se observan durante el lavado y lisis artroscópico, para el tratamiento de pacientes con movimientos mandibulares limitados. El término fue introducido por vez primera por Dorrit Nitzan, con su experiencia y éxito en el manejo del compartimiento articular superior de la ATM.

El lavado del espacio articular superior de pacientes con limitación a la abertu-
ISSN: 1560-9111

Caso Clí́nico

\section{José Cornejo Salazar ${ }^{1}$ Lizardo Sáenz Quiroz ${ }^{1}$ Elmo Palacios Alva ${ }^{1}$ María Vázquez Vazquez²}

1 Profesor Principal del Departamento Médico Quirúrgico de la F. Odontología UNMSM

Cirujano dentista, egresado UNMSM

Correspondencia:

Mg. C.D. José Luis Cornejo Salazar Facultad de Odontología

Universidad Nacional Mayor de San Marcos A. Germán Amézaga s/n Lima - Perú Correo: jcornejos@hotmail.com ra bucal y con desplazamiento anterior y medial del disco sin reducción se realiza con numerosos agentes como: soluciones de Lactato de Ringer, cloruro de $\mathrm{Na}$, esteroides como la triamcinolona o betametasona, agentes anestésicos (lidocaína, mepivacaína) opiáceos, el hialuronato de sodio, entre otros, con diferentes concentraciones. ${ }^{8}$ En nuestra experiencia, nosotros preferimos usar el cloruro de $\mathrm{Na}$.

David Frost sugiere que la indicación mayor para el uso de la artrocentesis es la limitación abertura mandibular, debido a un desplazamiento antero medial del disco articular sin reducción, así como a la hipomovilidad debida a una restricción en la traslación condilar9. Este proceso denominado "fenómeno de disco anclado" constituyó un concepto nuevo introducido tambien por Nitzan, quien, evaluando gran cantidad de casos que presentaban bloqueo mandibular, plantea su patogénesis debido a una adherencia abrupta del disco a la fosa asociada a una alteración de la lubricación normal articular, adoptando un carácter intermitente, con una activación secundaria de especies oxidativas y degradación del ácido hialurónico; esto genera fricción entre el disco y 
los componentes óseos articulares, con la subsiguiente deformidad discal y su desplazamiento. ${ }^{10}$

\section{Objetivo general}

Evaluar clínicamente la eficacia de la artrocentesis temporo-mandibular en el tratamiento de alteraciones de la relación condilo-disco que ocasiona bloqueo antero medial y como consecuencia limitación de la abertura bucal utilizando para el tratamiento un nuevo dispositivo de una sola punción.

\section{CASO CLÍNICO}

\section{Paciente y método}

Paciente mujer de 27 años, acude al Servicio de Cirugía Bucal y Maxilofacial de la Facultad de Odontología de la Universidad Nacional Mayor de San Marcos, con dolor orofacial agudo en articulación temporomandibular y limitación de abertura bucal desde hace 25 días, condicion que dificulta masticación y afecta calidad de vida. Ha tenido atención en varios consultorios médicos y dentales recibiendo tratamiento farmacológico que alivia parcialmente el dolor; trae radiografía panorámica no contributoria, estructuras maxilares y dentarias, dentro de límites normales.

\section{Diagnóstico}

Paciente adolorida refiere inicio brusco de condición que limita abertura bucal; no presenta antecedentes de enfermedad sistémica ni de traumatismo máxilofacial reciente; manifiesta haber tenido chasquido articular desde hace más o menos 10 años y que este cursaba con molestias leves.

A la exploracion clínica de músculos masticatorios, no contracturas ni puntos de dolor referido, dolor intenso en zona retrodiscal de ATM. Abertura bucal interincisiva sin dolor de $15 \mathrm{~mm}$, al intentar abertura forzada se incrementa dolor. Cavidad bucal con mucosa oral, humeda, elástica no congestiva, sistema dentario en buen estado de conservación e higiene.

\section{Impresión diagnóstica}

Síndrome de disco fijo o anclado que ocasiona bloqueo de abertura bucal y dolor articular por presión en la zona retrodiscal temporomandibular.
Se explica a la paciente posibilidad de tratamiento con procedimiento mínimamente invasivo; y luego del consentimiento informado, se realiza la artrocentesis con un nuevo dispositivo de una sola punción.

\section{Plan de tratamiento}

Se realiza en la Clínica Universitaria, bajo anestesia local infiltrativa, seleccionando al paciente con los siguientes criterios:

\section{Criterios de inclusión}

- Trastorno temporo-mandibulares que después de 21 días se muestren refractarios al tratamiento convencional (farmacoterapia, terapia física y terapia oclusal) y persista limitación de abertura con dolor persistente.

- Respuesta positiva al consentimiento informado.

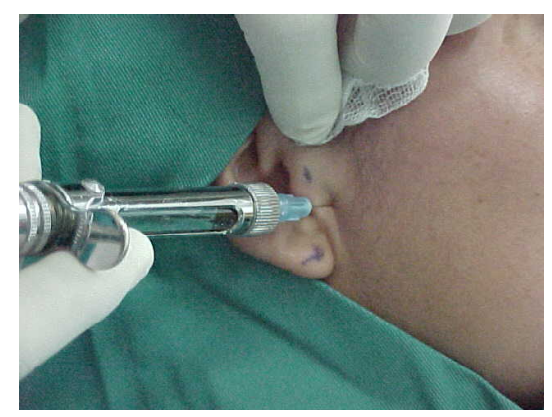

Fig. 1. Colocación de anestesia local.

\section{Criterios de exclusión}

- Trastorno témporomandibulares con afecciones óseas, metabólicas o inmunológicas de repercusión en el metabolismo osteo-articular.

- Paciente con procedimiento quirúrgico articular previo o que presente lesiones sépticas o inflamatoria en su proximidad temporomandibular.

- Pacientes con estado psicológico alterado no compensado.

\section{Descripción del procedimiento}

1. Posición del paciente. Paciente sentado reclinado con angulación de 120 grados con rotación cefálica hacia articulación no afectada.

2. Antisepsia y delimitación del campo operatorio. Se traza una línea del canto externo del ojo hacia el punto medio del tragus, mediante palpación manual el compartimiento articular superior.

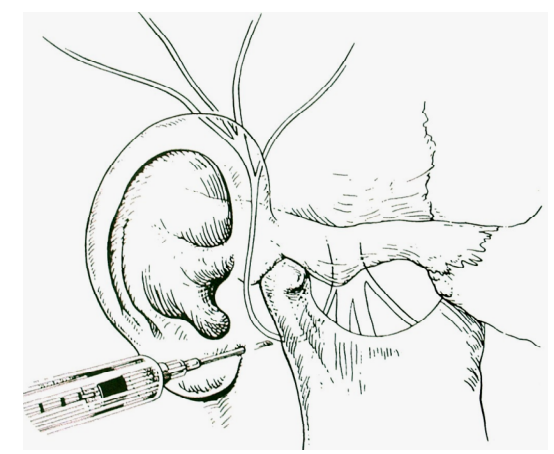

Fig 2. Lavaje articular.

Anestesia local: Se infiltra articaína con epinefrina para bloquear el nervio auriculotemporal.

3. Lavado articular propiamente dicho. Tras la colocación del dispositivo con aguja de doble entrada en el sitio de punción previamente definido, se instilan bajo presión la solución para el lavado, depositando previamente $2 \mathrm{ml}$ para comprobar que se halla en espacio articular superior y favoreciendo su recolección; después de comprobado, se procede a instilar $30 \mathrm{ml}$ de la solución de cloruro de $\mathrm{Na}$ preparada para el lavado; se realizará con presión controlada en jeringa con tiempo de instilación variable.

4. En el post operatorio inmediato, se pide al paciente que abra la boca y realice fisioterapia inmediata: indicándole que realice abertura y cierre mandibular y utilizando dispositivos coadyuvantes por 48 horas a 5 días, acudir a consulta para seguimiento en 72 horas.

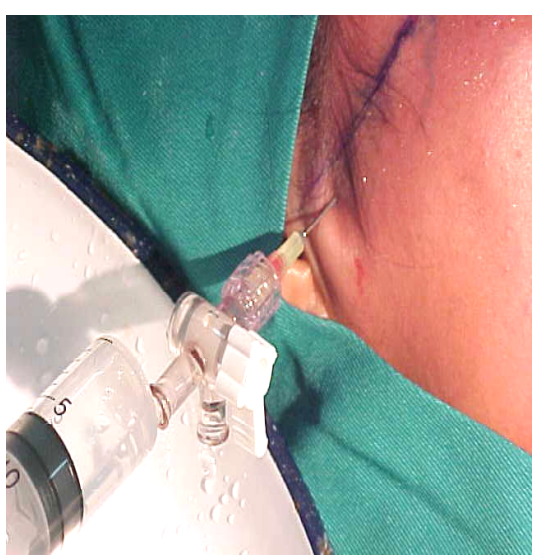

Fig 3. Con dispositivo de una sola punción.

\section{Material e instrumental empleado en} la artrocentesis

- Jeringa tipo carpule

- Dos carpules de anestesia dental de articaina con epinefrina

- Jeringa tipo Luer descartable de 20 $\mathrm{ml}$ 


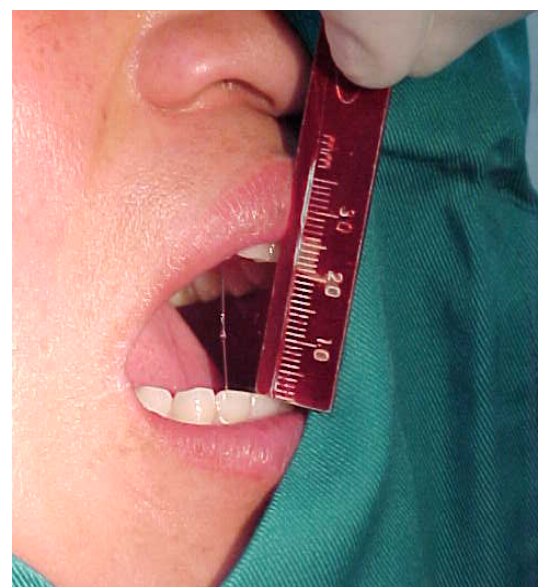

Fig 4. Abertura progresiva $23 \mathrm{~mm}$.

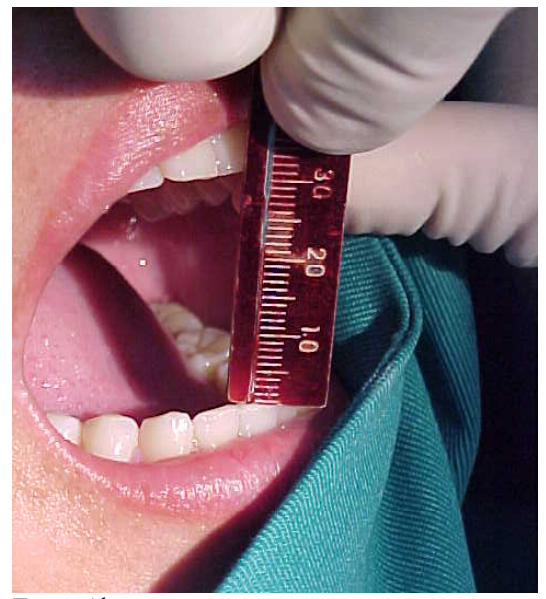

Fig 5. Abertura progresiva $33 \mathrm{~mm}$.

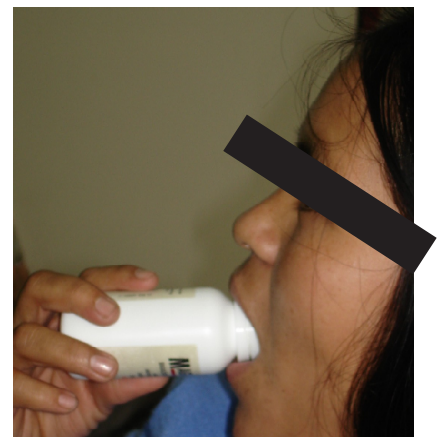

Fig 6. Dispositivo para fisioterapia que ayuda abertura bucal.

- dispositivo de doble entrada

- Una riñonera.

\section{Resultado}

Fue satisfactorio para la paciente; logrando abrir la boca a más de $35 \mathrm{~mm}$ de distancia interincisal luego del procedimiento; fig. 5 esta abertura se fue incrementando con los ejercicios recomendados, disminuyó el dolor orofacial y pudo reintegrarse rápidamente a sus actividades cotidianas. (Fig. 7)

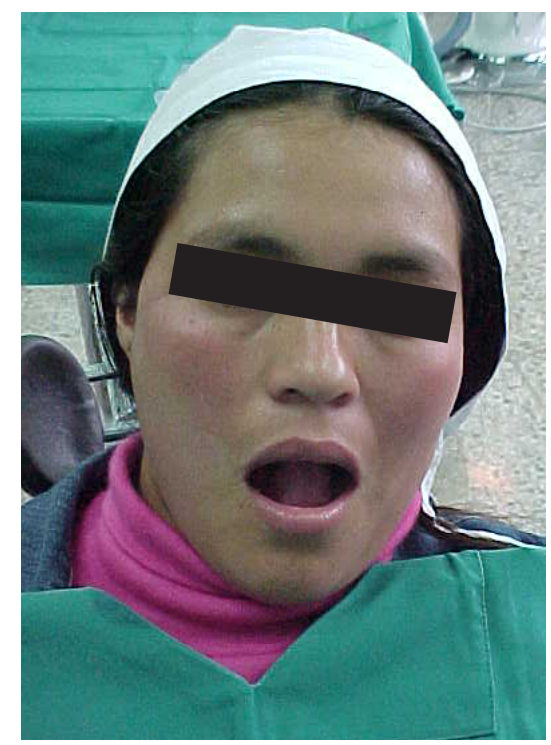

Fig 7. Paciente después artrocentesis.

\section{Análisis y Discusión}

El diagnóstico del síndrome de disco fijo, se fundamenta en la historia y exploración clínica, el tratamiento minimamente invasivo tiene indicaciones precisas: cuando no cede dolor orofacial y persiste bloqueo de la abertura bucal. ${ }^{11}$

La artrocentesis en ATM es un procedimiento mínimamente invasivo que permite, mediante un lavado del espacio articular superior, la aplicación de una presión hidráulica para liberar el disco articular de adherencias, bandas de tejido fibroso que se forman entre el disco articular y la cápsula, lo que impide el libre movimiento del disco. Además de eliminar mediadores químicos que acentúan los procesos inflamatorios articulares y su complejo sindrómico potencializadores de los procesos degenerativos articulares. ${ }^{12-13}$

Las concentraciones de bradicininas, interleucin-6 y proteínas, mediadores del proceso inflamatorio reducen considerablemente su presencia luego del lavado, lo que mejora eficazmente el síntoma dolor y el cuadro inflamatorio articular. Quinn y Bazan reportan una correlación positiva entre la presencia de prostaglandina E2 y leucotrienos en fluido sinovial y la presencia de sinovitis aguda. ${ }^{14-15}$

El valor promedio de máxima abertura bucal sin dolor de $15 \mathrm{~mm}$ pretratamiento, asociado a la falta de traslación condilar por el anclaje anterior del disco, consiguiéndose un valor inmediato resultante superior a los $(35 \mathrm{~mm})$.

La reducción del valor promedio del síntoma dolor en la escala visual analógica de 9,75 a 5,5 (más de 4 unidades) a las 72 horas, resultó un valor superior a la reducción de dolor para ese tiempo evolutivo reportada por Alpaslan, Sanromán y Dolwick. ${ }^{16}$

\section{Conclusiones}

- La artrocentesis es un procedimiento mínimamente invasivo que mejora la abertura bucal y alivia el dolor en el bloqueo discal anteromedial de pacientes refractarios a tratamiento conservador.

- La artrocentesis permite eliminar sustancias nocivas y mejora la lubricación de la articulación temporomandibular realizandose en una sesión; con los cuidados y el entrenamiento necesario.

- Utilizando este dispositivo, no se requiere la inserción de dos agujas lo que facilita el procedimiento siendo el edema periarticular postoperatorio un evento transitorio sin complicaciones.

\section{Referencias bibliográficas}

1. Murphy E, Uyanik J, Sanders B. Evaluation and treatment of temporomandibular disorders: considerations for the general dentist. Alpha Omegan, 2000; 93: 47.

2. Hellen Cordeiro CorrêaI et al. Joint disorder: nonreducing disc displacement with mouth opening limitation - report of a case Journal of Applied Oral Science. 2009; 17(4) vol.17 N.er 4 Bauru July\Aug. 2009.

3. Nitzan DW, Dolwick MF, Heft MW. Arthroscopic lavage and lysis of the temporomandibular Joint: A change in perspective. J Oral Maxillofac Surg 1990; 48; 798 - 8.

4. Nitzan DW. Friction and Adhesive Forces - Possible Underlying Causes for Temporomandibular Joint Internal Derangement. Cells Tisúes Organs 2003; 174: 6-16.

5. Crow et al. The utility of panoramic radiography in temporomandibular joint assessment. Dentomaxillofac Radiol. 2005; 34: 91-95.

6. Functional magnetic resonance imaging of temporomandibular joint. D Eberhard - Journal Clinical Radiology 2000; 47: 127-133.

7. Nishimura M, Segami N, Kaneyama $\mathrm{K}$ et al. Prognostic factors in arthrocentesis of the temporoman- 
dibular joint: Evaluation of 100 patients with internal derangement. J Oral Maxillofac Surg 2001; 59: 874.

8. Frost DE, Kendall BD. The use of arthrocentesis for treatment of the temporomandibular joint disorders. J Oral Maxillofac Surg 1999; 57: 583-587.

9. Morales Trejo B. Evaluación y conceptos de Artrocentesis. Rev A.D.M. 2003; 60(1): 25-28.

10. Alpaslan GH, Alpaslan C. Efficacy of temporomandibular joint arthrocentesis with and without injection of sodium hyaluronate in treatment of internal derangements. J Oral Maxillofac Surg 2001; 59(6): 613-8.
11. White RD. Arthroscopic lysis and lavage as the preferred treatment for internal derangements of the temporomandibularjoint. J Oral Maxillofac Surg 2001; 59: 313.

12. Nitzan DW, Dolwick MF, Martínez GA. Temporomandibular joint arthrocentesis: A simplified treatment for severe limited mouth opening. J Oral Maxillofac Surg 1991; 49: 1163-1167.

13. Murakami K. Fouryear follow-up study of temporomandibular joint arthroscopic surgery for advanced stage internal derangements. Journal of Oral and Maxillofacial Surgery 1996; 54: 285.

14. Kaneyama K, Segami N, Nishimura M, Suzuki T, Sato J. Importance of proinflammatory of cytokines in synovial fluid from 121 joints with temporomandibular disorders. $\mathrm{Br}$ J Oral Maxillofac Surg 2002; 40: 418-23.

15. Kaneyama K. The Ideal Lavage Volume for Removing Bradykinin, Interleukin-6, and Protein From the Temporomandibular Joint by Arthrocentesis. J Oral Maxillofac Surg, 2004; 62: 657-661.

16. Nitzan, D.W., Y. Etsion (Adhesive force - The underlying cause of the 'anchored disc phenomenon'. Int J Oral Maxillofac Surg 2002; 31(1): 94-99.

Fecha de recepción: 24-6-09

Fecha de aprobación: 10-06-10 